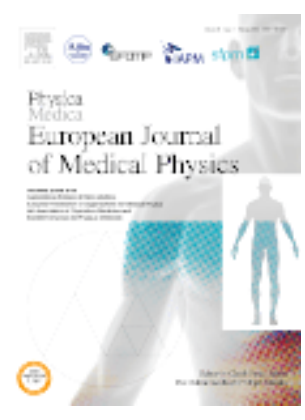

\title{
Comparison of different breast planning techniques and algorithms for radiation therapy treatment
}

- $\quad$ C. Borges $^{\mathrm{a}}, \mathbf{\Delta}, \nabla$,

- G. Cunha $^{b}$,

- I. Monteiro-Grilloc,

- $\quad$ P. Vaz ${ }^{d}$,

- N. Teixeira $^{b}$

- ${ }^{a}$ Medicalconsult SA, Campo Grande, $n^{\circ} 56-8^{\circ}$ A, 1700-093 Lisboa, Portugal

- ${ }^{b}$ Escola Superior de Tecnologias da Saúde, Avenida Dom João II, 1900-096 Lisboa, Portugal

- $\quad{ }^{\mathrm{C}}$ Departamento de Radioterapia, Hospital de Santa Maria, Centro Hospitalar Lisboa, Norte, EPE, Avenida Professor Egas Moniz, 1649-035 Lisboa, Portugal

- d Instituto Superior Técnico, Campus Tecnológico e Nuclear, Unidade de Protecção e Segurança Radiológica, Estrada Nacional 10 (ao km 139,7), 2695066 Bobadela LRS, Portugal

\section{Abstract}

This work aims at investigating the impact of treating breast cancer using different radiation therapy (RT) techniques - forwardly-planned intensity-modulated, f-IMRT, inversely-planned IMRT and dynamic conformal arc (DCART) RT - and their effects on the whole-breast irradiation and in the undesirable irradiation of the surrounding healthy tissues. Two algorithms of iPlan BrainLAB treatment planning system were compared: Pencil Beam Convolution (PBC) and commercial Monte Carlo (iMC).

Seven left-sided breast patients submitted to breast-conserving surgery were enrolled in the study. For each patient, four RT techniques - f-IMRT, IMRT using 2-fields and 5fields (IMRT2 and IMRT5, respectively) and DCART - were applied. The dose distributions in the planned target volume (PTV) and the dose to the organs at risk 
(OAR) were compared analyzing dose-volume histograms; further statistical analysis was performed using IBM SPSS v20 software.

For PBC, all techniques provided adequate coverage of the PTV. However, statistically significant dose differences were observed between the techniques, in the PTV, OAR and also in the pattern of dose distribution spreading into normal tissues. IMRT5 and DCART spread low doses into greater volumes of normal tissue, right breast, right lung and heart than tangential techniques. However, IMRT5 plans improved distributions for the PTV, exhibiting better conformity and homogeneity in target and reduced high dose percentages in ipsilateral OAR. DCART did not present advantages over any of the techniques investigated. Differences were also found comparing the calculation algorithms: PBC estimated higher doses for the PTV, ipsilateral lung and heart than the iMC algorithm predicted.

\section{Keywords}

- Breast radiotherapy;

- Irradiation techniques;

- Calculation;

- Algorithms

Corresponding author. Av. Embaixador Assis Chateaubriand, n2 2 D, 2780-196 Oeiras, Portugal. Tel.: +351 927054182.

Copyright (C 2013 Associazione Italiana di Fisica Medica. Published by Associazione Italiana di Fisica in Medicina All rights reserved. 